\title{
A.P.O. Rules are Asymptotically non Deficient for Estimation with Squared Error Loss
}

\author{
Michael Woodroofe* \\ The University of Michigan, Dept. of Statistics, Ann Arbor, MI 48109, USA
}

\begin{abstract}
Summary. The problem considered is sequential estimation of the mean $\theta$ of a one-parameter exponential family of distributions with squared error loss for estimation error and a cost $c>0$ for each of an i.i.d. sequence of potential observations $X_{1}, X_{2}, \ldots$ A Bayesian approach is adopted, and natural conjugate prior distributions are assumed. For this problem, the asymptotically pointwise optimal (A.P.O.) procedure continues sampling until the posterior variance of $\theta$ is less than $c\left(r_{0}+n\right)$, where $n$ is the sample size and $r_{0}$ is the fictitous sample size implicit in the conjugate prior distribution. It is known that the A.P.O. procedure is Bayes risk efficient, under mild integrability conditions. In fact, the Bayes risk of both the optimal and A.P.O. procedures are asymptotic to $2 V_{0} \sqrt{c}$, as $c \rightarrow 0$, where $V_{0}$ is the prior expectation of the standard deviation of $X_{1}$, given $\theta$. Here the A.P.O. rule is shown to be asymptotically non-deficient, under stronger regularity conditions: that is, the difference between the Bayes risk of the A.P.O. rule and the Bayes risk of the optimal procedure is of smaller order of magnitude than $c$, the cost of a single observation, as $c \rightarrow 0$. The result is illustrated in the exponential and Bernoulli cases, and extended to the case of a normal distribution with both the mean and variance unknown.
\end{abstract}

\section{Conjugate Prior Distributions for Exponential Families}

Let $\Omega$ be an interval and let $F_{\omega}, \omega \in \Omega$, denote a non degenerate exponential family of probability distributions on the Borel sets of $(-\infty, \infty)$ : that is, suppose that

$$
d F_{\omega}(x)=\exp \{\omega x-\psi(\omega)\} d \lambda(x) \quad+\infty<x<\infty, \omega \in \Omega .
$$

Here $\Omega$ denotes the natural parameter space of the family; and $\Omega$ is assumed to be open, say $\Omega=(\underline{\omega}, \bar{\omega})$, where $-\infty \leqq \underline{\omega}<\bar{\omega} \leqq \infty$. The distributions $F_{\omega}, \omega \in \Omega$,

* Research supported by the National Science Foundation Grant NSF-MCS-78-02468 
are mutually absolutely continuous. Their common closed convex support is denoted by $X$; and the interior of $X$ is denoted by $X^{0}$. It is well known that if $X$ is a random variable with distribution $F_{\omega}$, where $\omega \in \Omega$, then the mean and variance of $X$ are $E_{\omega}(X)=\psi^{\prime}(\omega)$ and $D_{\omega}(X)=\psi^{\prime \prime}(\omega)$. The mean is of special interest here and is denoted by

$$
\theta=\psi^{\prime}(\omega)
$$

throughout. It is easily seen that $\theta=\psi^{\prime}$ is a strictly increasing function from $\Omega$ onto $X^{0}$, so the distributions $F_{\omega}, \omega \in \Omega$, could be parameterized by $\theta$ as well as $\omega$. See Lehmann (1959, Sect. 2.7) for the elementary properties of exponential families, and Barndorff-Nielson (1978) for a more detailed discussion.

Diaconis and Ylvisaker (1979) have given an interesting characterization of conjugate prior distributions for exponential families. If $r_{0}>0$ and $\mu_{0} \in X^{0}$, then

so,

$$
0<c\left(r_{0}, \mu_{0}\right)=\int_{\Omega} \exp \left\{r_{0} \mu_{0} \omega-r_{0} \psi(\omega)\right\} d \omega<\infty
$$

$$
\xi_{0}(\omega)=\left(1 / c\left(r_{0}, \mu^{0}\right)\right) \exp \left\{r_{0} \mu_{0} \omega-r_{0} \psi(\omega)\right\}, \quad \omega \in \Omega,
$$

defines a density w.r.t. Lebesgue measure on the Borel sets of $\Omega$. The corresponding distribution is denoted by $\pi_{0}$, so that $d \pi_{0}(\omega)=\xi_{0}(\omega) d \omega, \omega \in \Omega$. It is then the case that

$$
E(\theta)=\int_{\Omega} \psi^{\prime} d \pi_{0}=\mu_{0}
$$

When applied to the posterior distribution of $\omega$, given an observation $X \sim F_{\omega}$, Eq. (1) asserts that the posterior expectation $E(\theta \mid X)$ is linear in $X$; and this property is characteristic of prior distributions of the form $\pi_{0}$, under some additional conditions on $X$.

The following simple extension of $(1)$ is central to the analysis of asymptotically pointwise optimal rules: if

then

$$
E\left[\psi^{\prime \prime}(\omega)\right]=\int_{\Omega} \psi^{\prime \prime} d \pi_{0}<\infty
$$

$$
E\left[\left(\theta-\mu_{0}\right)^{2}\right]=r_{0}^{-1} E\left[\psi^{\prime \prime}(\omega)\right] .
$$

Equation (2) follows from Lemma 1, below, applied to $g(\omega)=\left(\theta-\mu_{0}\right), \omega \in \Omega$.

Lemma 1. If $g$ is a continuously differentiable function on $\Omega$ for which

then

$$
\int_{\Omega}\left|g^{\prime}\right| d \pi_{0}<\infty
$$

$$
\int_{\Omega} g \xi_{0}^{\prime} d \omega=-\int_{\Omega} g^{\prime} \xi_{0} d \omega .
$$

If $\lim \sup g(\omega)<0<\lim \inf g(\omega)$, then $\left|g^{\prime}\right|$ may be replaced by $g_{+}^{\prime}=\max \left\{0, g^{\prime}\right\}$ in condition (3).

Proof. It suffices to show that $\left(g \xi_{0}\right)(\omega) \rightarrow 0$ as either $\omega \rightarrow \underline{\omega}$ or $\omega \rightarrow \bar{\omega}$. Diaconis and Ylvisaker (1979) show that $\xi_{0}(\omega) \rightarrow 0$ as $\omega \rightarrow \underline{\omega}$ or $\omega \rightarrow \bar{\omega}$. This fact is used. 
Let $\hat{\omega}_{0}$ be the prior mode - that is $\psi^{\prime}\left(\hat{\omega}_{0}\right)=\mu_{0}$; and observe that $\xi_{0}(\omega)$ is increasing in $\omega<\hat{\omega}_{0}$ and decreasing in $\omega>\hat{\omega}_{0}$. If $\hat{\omega}_{0}<\omega_{0}<\omega_{1}<\bar{\omega}$, then

$$
\begin{aligned}
\left|g\left(\omega_{1}\right)\right| \xi\left(\omega_{1}\right) & \leqq\left\{\left|g\left(\omega_{0}\right)\right|+\int_{\omega_{0}}^{\omega_{1}}\left|g^{\prime}(\omega)\right| d \omega\right\} \xi_{0}\left(\omega_{1}\right) \\
& \leqq\left|g\left(\omega_{0}\right)\right| \xi_{0}\left(\omega_{1}\right)+\int_{\omega_{0}}^{\omega_{1}}\left|g^{\prime}(\omega)\right| \xi_{0}(\omega) d \omega .
\end{aligned}
$$

So,

$$
\limsup _{\omega \rightarrow \bar{\omega}}|g(\omega)| \xi_{0}(\omega) \leqq \int_{\omega_{0}}^{\bar{\omega}}\left|g^{\prime}(\omega)\right| \xi_{0}(\omega) d \omega
$$

which tends to zero as $\omega_{0} \rightarrow \bar{\omega}$. The behavior of $g \xi_{0}$ at the lower end-point may be analyzed similarly.

If $g(\omega)>0$ for $\omega$ in some neighborhood of $\bar{\omega}$ and only $g_{+}^{\prime}$ is assumed to be integrable w.r.t. $\pi_{0}$, then (4) holds with $\left|g^{\prime}\right|$ replaced by $g_{+}^{\prime}$, provided that $\omega_{0}$ is sufficiently close to $\bar{\omega}$. The remainder of the proof proceeds as above.

\section{Sequential Estimation}

In this section and the next, $\pi_{0}$ denotes a conjugate prior distribution with $r_{0}>0$ and $\mu_{0} \in X^{0}$; and $\omega$ and $X_{1}, X_{2}, \ldots$ are random variables, defined on some probability space $(\mathscr{P}, \mathscr{A}, P)$, for which $\omega \sim \pi_{0}$ and $X_{1}, X_{2}, \ldots$ are conditionally i.i.d. with common distribution $F_{\omega}$, given $\omega$. Let $\mathscr{A}_{0}=\{\phi, \mathscr{A}\}$ be the trivial sigma-algebra and let $\mathscr{A}_{n}=\sigma\left\{X_{1}, \ldots, X_{n}\right\}$ be the sigma-algebra generated by $X_{1}, \ldots, X_{n}$ for $n \geqq 1$. Then the conditional, or posterior, distribution of $\omega$ given $\mathscr{A}_{n}$ is

where

$$
d \pi_{n}(\omega)=\left(1 / c\left(r_{n}, \mu_{n}\right)\right) \exp \left\{r_{n} \mu_{n} \omega-r_{n} \psi(\omega)\right\} d \omega, \quad \omega \in \Omega,
$$

$$
r_{n}=r_{0}+n \text { and } \mu_{n}=\left(r_{0} \mu_{0}+S_{n}\right) / r_{n}
$$

with $S_{n}=X_{1}+\ldots+X_{n}$ for $n \geqq 1$.

Recall that a stopping time is a random variable $t$ which takes the values $0,1,2, \ldots$ and $\infty$ and has the properties $P\{t<\infty\}=1$ and $\{t=n\} \in \mathscr{A}_{n}$ for all $n \geqq 0$. The sigma-algebra $\mathscr{A}_{1}$ of events which occur prior to a stopping time $t$ is the class of all $A \in \mathscr{A}$ for which $A\{t=n\} \in \mathscr{A}_{n}$ for all $n \geqq 0$. Then, it is easily seen that the conditional distribution of $\omega$ given $\mathscr{A}_{t}$ is simply $\pi_{t}$ on $\{t<\infty\}$.

Now suppose that $E\left[\psi^{\prime \prime}(\omega)\right]<\infty$, so that $\theta$ has a finite variance, and consider the problem of sequentially estimating $\theta$ with squared error loss for estimation error and a cost $c>0$ for each observation $X_{1}, X_{2}, \ldots$ The Bayesian sequential decision problem is then to find a stopping time $t$ and an $\mathscr{A}_{t}$ measurable function $\hat{\theta}_{t}=\hat{\theta}_{t}\left(X_{1}, \ldots, X_{t}\right)$ for which

$$
\text { Bayes risk }\left(t, \hat{\theta}_{t}\right)=E\left\{\left(\hat{\theta}_{t}-\theta\right)^{2}+c t\right\}
$$

is minimized. It is well known that for any stopping time $t$, the Bayes risk is minimized by letting $\hat{\theta}_{t}=\mu_{t}=E\left(\theta \mid \mathscr{A}_{t}\right)$. See, for example, Ferguson (1967, pp. 314-15). Moreover, it follows from (2) that, for any stopping time $t$, 
where

$$
E\left\{\left(\theta-\mu_{t}\right)^{2}\right\}=E\left\{E\left[\left(\theta-\mu_{t}\right)^{2} \mid \mathscr{A}_{t}\right]\right\}=E\left\{r_{t}^{-1} U_{t}\right\}
$$

$$
U_{n}=E\left[\psi^{\prime \prime}(\omega) \mid \mathscr{A}_{n}\right]=\int_{\Omega} \psi^{\prime \prime} d \pi_{n}, \quad n \geqq 0 .
$$

It is relevant that $U_{n}, n \geqq 0$, is a uniformly integrable martingale for which $U_{n} \rightarrow \psi^{\prime \prime}(\omega)$ w.p.l as $n \rightarrow \infty$. Next let

Then

$$
L_{n}(c)=r_{n}^{-1} U_{n}+c r_{n}, \quad n \geqq 0, c>0 .
$$

$$
\text { Bayes risk }\left(t, \mu_{t}\right)=E\left\{L_{t}(c)\right\}-c r_{0}, \quad c>0,
$$

for any stopping time $t$; so, minimizing the Bayes risk is equivalent to minimizing $E\left\{L_{t}(c)\right\}$ by choice of $t$.

It follows from Theorems 4.4 and 4.5 of Chow, Robbins and Siegmund (1970) that an optimal stopping time, one which minimizes $E\left\{L_{t}(c)\right\}$, exists for each $c>0$. In principle, the optimal stopping time may be computed by backward induction and a limiting operation; but the exact determination of the optimal stopping time appears to be a formidable task, in practice. Bickel and Yahav $(1967,1968,1969$ a) have developed an interesting class of asymptotic solutions to the minimization problem, as $c \rightarrow 0$. They describe methods for finding stopping times $t=t_{c}$ which are asymptotically pointwise optimal (A.P.O.) in the sense that

$$
L_{t}(c) \sim \inf _{n} L_{n}(c) \sim 2 \sqrt{\left[c \psi^{\prime \prime}(\omega)\right]}
$$

w.p.l as $c \rightarrow 0$. Replacing $n$ by $r_{n}$ in Bickel and Yahav's (1967) A.P.O. rule suggests the stopping times

$$
t=t_{c}=\inf \left\{n \geqq 0: U_{n}<c r_{n}^{2}\right\}, \quad c>0 .
$$

In fact, a careful reading of their derivation yields the following: if $s=s_{c}$ are stopping times, then, as $c \rightarrow 0$,

$$
L_{s}(c) \sim \inf _{n} L_{n}(c) \quad \text { iff } s_{c} \sim t_{c}
$$

except for a set of probability zero.

In the next section, the A.P.O. rule $t=t_{c}$ of (6) is shown to be asymptotically optimal in the following strong sense: let

$$
\rho_{0}(c)=\inf _{s} E\left[L_{s}(c)\right], \quad c>0,
$$

where the infimum extends over all stopping times $s$; then $E\left\{L_{t}(c)\right\}=\rho_{0}(c)$ $+o(c)$, as $c \rightarrow 0$, under a mild integrability condition. That is, the A.P.O. rule $t$ is asymptotically non-deficient in the sense that the additional cost which results from using the sub-optimal procedure $t$ is of smaller order of magnitude than the cost of a single observation. Bickel and Yahav (1968) have shown that $E\left\{L_{t}(c)\right\} / \rho_{0}(c) \rightarrow 1$ in a context which is more general than the present one; and Alvo (1977) has shown that the deficiency of a closely related procedure is of 
order $c$, under conditions which are related to the present ones but are not strictly comparable. The representation (6) for the Bayes risk is implicit in his examples. Shapiro and Wardrop (1980a) have used a representation which is similar to (6) in their study of the myopic procedure. They consider only the term of order $\sqrt{c}$ in the asymptotic expansion, however. Finally, Vardi (1979) has recently given an interesting discussion of Bayes procedures for diffuse priors.

\section{Asymptotic Optimality}

The proof of asymptotic optimality depends on the simple identity,

$$
L_{n}(c)=r_{n}^{-1} U_{n}+c r_{n}=2 \sqrt{\left(c \overline{U_{n}}\right)}+r_{n}^{-1}\left[\sqrt{U_{n}}-r_{n} \sqrt{c}\right]^{2}
$$

for $n \geqq 0$ and $c>0$. It is shown below that the last term on the right side of (9) contributes only $o(c)$ to the expected loss for both the optimal and A.P.O. rules. To understand the first, let

$$
V_{n}=E\left[\sqrt{\psi^{\prime \prime}(\omega)} \mid \mathscr{A}_{n}\right] \text { and } W_{n}=U_{n}-V_{n}^{2}, \quad n \geqq 0 .
$$

Then $V_{n}, n \geqq 0$, is a uniformly integrable martingale; so,

$$
E\left[V_{s}\right]=V_{0}=\int_{\Omega} \sqrt{\psi^{\prime \prime}} d \pi_{0}
$$

for every stopping time $s$, by the optional stopping theorem. (See, for example, Theorem 2.3 of Chow, Robbins, and Siegmund (1970).) Observe that $W_{n}$ is the posterior variance of $\sqrt{\psi^{\prime \prime}(\omega)}$ given $\mathscr{A}_{n}$ and that $0<W_{n} \rightarrow \psi^{\prime \prime}(\omega)-\psi^{\prime \prime}(\omega)=0$ w.p.1 as $n \rightarrow \infty$. Now

so

$$
\sqrt{U_{n}}=V_{n}+\frac{W_{n}}{\sqrt{\bar{U}_{n}+V_{n}}}, \quad n \geqq 0,
$$

$$
E\left[\sqrt{U_{s}}\right]=V_{0}+E\left[\frac{W_{s}}{\sqrt{U_{s}}+V_{s}}\right]
$$

for any stopping time $s$. Clearly, an expansion for $E\left[W_{s} /\left(\sqrt{U_{s}}+V_{s}\right)\right]$ is needed.

In the next lemma, the following result is used: let $\hat{\omega}_{n}$ denote the posterior mode, so that $\hat{\omega}_{n}$ solves the equation $\psi^{\prime}\left(\hat{\omega}_{n}\right)=\mu_{n}$; as $n \rightarrow \infty$, the posterior distribution of $\sqrt{\left[r_{n} \psi^{\prime \prime}\left(\hat{\omega}_{n}\right)\right]} \cdot\left(\omega-\hat{\omega}_{n}\right)$ converges to the standard normal distribution w.p.1. See Bickel and Yahav $(1969 \mathrm{~b})$ for this result in a more general context; or see Johnson (1970) for a more detailed result for exponential families.

Lemma 2. Let $\gamma(\theta)=\sqrt{\psi^{\prime \prime}(\omega)}$, where $\theta=\psi^{\prime}(\omega)$; and suppose that

$$
\int_{\Omega} \gamma^{\prime}(\theta)^{2} \psi^{\prime \prime} d \pi_{0}<\infty
$$


Then

and

$$
r_{n} W_{n} \leqq E\left[\gamma^{\prime}(\theta)^{2} \psi^{\prime \prime}(\omega) \mid \mathscr{A}_{n}\right], \quad \text { w.p. } 1, n \geqq 1 \text {, }
$$

$$
r_{n} W_{n} \rightarrow \gamma^{\prime}(\theta)^{2} \psi^{\prime \prime}(\omega) \quad \text { w.p.l, } n \rightarrow \infty .
$$

Proof. For fixed $X_{1}, \ldots, X_{n}$, define a function $g$ on $\Omega$ by $g\left(\hat{\omega}_{n}\right)=0$ and $g(\omega)$ $=\left[\gamma(\theta)-\gamma\left(\mu_{n}\right)\right]^{2} /\left(\theta-\mu_{n}\right)$ for $\omega \neq \hat{\omega}_{n}$. Then

$$
r_{n} W_{n} \leqq r_{n} \int_{\Omega}\left[\gamma(\theta)-\gamma\left(\mu_{n}\right)\right]^{2} d \pi_{n}=-\int_{\Omega} g \xi_{n}^{\prime} d \omega
$$

where $\xi_{n}$ denotes the posterior density, $\xi_{n}(\omega)=\left(1 / c\left(r_{n}, \mu_{n}\right)\right) \exp \left\{r_{n} \mu_{n} \omega-r_{n} \psi(\omega)\right\}$, $\omega \in \Omega$. Now

and

$$
\begin{aligned}
\frac{d}{d \theta} g(\omega) & =2 \gamma^{\prime}(\theta)\left[\frac{\gamma(\theta)-\gamma\left(\mu_{n}\right)}{\theta-\mu_{n}}\right]-\left[\frac{\gamma(\theta)-\gamma\left(\mu_{n}\right)}{\theta-\mu_{n}}\right]^{2} \\
& =\gamma^{\prime}(\theta)^{2}-\left\{\gamma^{\prime}(\theta)-\left[\frac{\gamma(\theta)-\gamma\left(\mu_{n}\right)}{\theta-\mu_{n}}\right]\right\}^{2} \leqq \gamma^{\prime}(\theta)^{2}
\end{aligned}
$$

$$
g^{\prime}(\omega)=\frac{d}{d \theta} g(\omega) \cdot \psi^{\prime \prime}(\omega) \leqq \gamma^{\prime}(\theta)^{2} \psi^{\prime \prime}(\omega), \quad \omega \neq \hat{\omega}_{n}
$$

Thus, $g^{\prime}(\omega)$ is bounded above by a function which is integrable w.r.t. $\pi_{0}$. Since $g(\omega)<0$ for $\omega<\hat{\omega}_{n}$ and $g(\omega)>0$ for $\omega>\hat{\omega}_{n}$, Lemma 1 yields

$$
\begin{aligned}
-\int_{\Omega} g \xi_{n}^{\prime} d \omega & =\int_{\omega} g^{\prime} \xi_{n} d \omega \\
& \leqq \int_{\Omega} \gamma^{\prime}(\theta)^{2} \psi^{\prime \prime} d \pi_{n}=E\left[\gamma^{\prime}(\theta)^{2} \psi^{\prime \prime}(\omega) \mid \mathscr{A}_{n}\right], \quad n \geqq 1 .
\end{aligned}
$$

This establishes the first assertion of the lemma.

To establish the second assertion, first observe that $E\left[\gamma^{\prime}(\theta) \psi^{\prime \prime}(\omega) \mid \mathscr{A}_{n}\right]$ $\rightarrow \gamma^{\prime}(\theta)^{2} \psi^{\prime \prime}(\omega)$ w.p.l as $n \rightarrow \infty$ by the martingale convergence theorem; so, $\limsup r_{n} W_{n} \leqq \gamma^{\prime}(\theta)^{2} \psi^{\prime \prime}(\omega)$ w.p.l as $n \rightarrow \infty$. The reverse inequality follows from the fact that the posterior distribution of $\omega$ is asymptotically normal with mean $\hat{\omega}_{n}$ and variance $1 / r_{n} \psi^{\prime \prime}\left(\hat{\omega}_{n}\right)$ and Fatou's Lemma.

Lemma 3. Suppose that

$$
\int_{\Omega} \gamma^{\prime}(\theta)^{4 \alpha} \psi^{\prime \prime} d \pi_{0}<\infty>\int_{\Omega}\left[\psi^{\prime \prime}+\left(1 / \psi^{\prime \prime}\right)\right] d \pi_{0}
$$

for some $\alpha>1$. If $s=s_{c}$ are any stopping times for which $\sqrt{c} \cdot s_{c} \rightarrow \sqrt{\psi^{\prime \prime}(\omega)}$ w.p.l as $c \rightarrow 0$, then

$$
\liminf _{c \rightarrow 0} c^{-\frac{1}{2}} E\left[\frac{W_{s}}{\sqrt{U_{s}}+V_{s}}\right] \geqq \frac{1}{2} \int_{\Omega} \gamma^{\prime}(\theta)^{2} d \pi_{0} ;
$$

and if $s_{c}=t_{c}$ is the A.P.O. rule, then the limit exists and there is equality in (12). 
Proof. If $s=s_{c}$ are stopping times for which $\sqrt{c} \cdot s_{c} \rightarrow \sqrt{\psi^{\prime \prime}(\omega)}$ w.p.l, then

$$
c^{-\frac{1}{2}}\left(\frac{W_{s}}{\sqrt{U_{s}+V_{s}}}\right)=\left(\frac{1}{\sqrt{c} \cdot r_{s}}\right)\left(\frac{r_{s} W_{s}}{\sqrt{U_{s}}+V_{s}}\right) \rightarrow \frac{\gamma^{\prime}(\theta)^{2} \psi^{\prime \prime}(\omega)}{2 \psi^{\prime \prime}(\omega)}=\frac{1}{2} \gamma^{\prime}(\theta)^{2}
$$

w.p.l as $c \rightarrow 0$; so (12) follows from Fatou's Lemma. Moreover, if $s=t$ is the A.P.O. rule, then $\sqrt{c} \cdot r_{t} \geqq \sqrt{U_{t}}$, so that

$$
\left(\frac{1}{\sqrt{c} \cdot r_{t}}\right)\left(\frac{r_{t} W_{t}}{\sqrt{U_{t}}+V_{t}}\right) \leqq \frac{r_{t} W_{t}}{U_{t}} \leqq \frac{E\left[\gamma^{\prime}(\theta)^{2} \psi^{\prime \prime}(\omega) \mid \mathscr{A}_{t}\right]}{E\left[\psi^{\prime \prime}(\omega) \mid \mathscr{A}_{t}\right]} .
$$

Thus, it suffices to show that the right side of (13) is uniformly integrable.

Uniform integrability is demonstrated by showing that the expected $\alpha^{\text {th }}$ power remains bounded. In the proof let

$$
Y=\psi^{\prime \prime}(\omega) / E\left[\psi^{\prime \prime}(\omega)\right] \text { and } Z=\gamma^{\prime}(\theta)^{2}
$$

and let $P^{\#}$ be the measure defined by $d P^{\#}=Y d P$. Then the quantity of interest may be written $E\left(Y Z \mid \mathscr{A}_{t}\right) / E\left(Y \mid \mathscr{A}_{t}\right)=E^{\#}\left(Z \mid \mathscr{A}_{t}\right)$; and

$$
\begin{aligned}
\int E^{\#}\left(Z \mid \mathscr{A}_{t}\right)^{\alpha} d P & =\int E^{\#}\left(Z \mid \mathscr{A}_{t}\right)^{\alpha} E^{\#}\left(1 / Y \mid \mathscr{A}_{t}\right) d P^{\#} \\
& \leqq \sqrt{\left[E^{\#}\left(Z^{2 \alpha}\right) E^{\#}\left(1 / Y^{2}\right)\right]}=\sqrt{\left[E\left(Y Z^{2 \alpha}\right) E(1 / Y)\right]},
\end{aligned}
$$

which is finite and independent of $c>0$. Uniform integrability follows.

The asymptotic behavior of $r_{t}^{-1}\left[r_{t} \sqrt{c}-\sqrt{U_{t}}\right]^{2}$ is considered next.

Lemma 4. Let

If

$$
Y_{n}=r_{n}\left[\sqrt{\left(\frac{U_{n-1}}{U_{n}}\right)}-1\right]_{+}^{2}, \quad n \geqq 0 .
$$

$$
E\left[\sup _{n \geqq 1} Y_{n}\right]<\infty
$$

then

$$
E\left[r_{t}^{-1}\left(r_{t} \sqrt{c}-\sqrt{U_{t}}\right)^{2}\right]=o(c), \quad \text { as } c \rightarrow 0 .
$$

Proof. Clearly, $t \geqq 1$ for sufficiently small $c>0$; and for such $c$, one has

and

$$
r_{t}^{-1}\left[r_{t} \sqrt{c}-\sqrt{U_{t}}\right]^{2} \leqq 2 r_{t}^{-1} c+2 r_{t}^{-1}\left[\sqrt{U_{t-1}}-\sqrt{U_{t}}\right]^{2}
$$

$$
r_{t}^{-1}\left[\sqrt{U_{t-1}}-\sqrt{U_{t}}\right]^{2}=r_{t}^{-2} U_{t} \cdot Y_{t} \leqq c Y_{t}
$$

by definition of the A.P.O. rule $t$. Thus, since $t \rightarrow \infty$ w.p.l as $c \rightarrow 0$, it suffices to show that $Y_{n} \rightarrow 0$ w.p.l as $n \rightarrow \infty$. The results of Johnson (1970) imply that $U_{n}$ $=\psi^{\prime \prime}\left(\hat{\omega}_{n}\right)+0(1 / n) \quad$ w.p.l; and $\psi^{\prime \prime}\left(\hat{\omega}_{n}\right)-\psi^{\prime \prime}\left(\hat{\omega}_{n-1}\right)=\gamma^{2}\left(X_{n}\right)-\gamma^{2}\left(X_{n-1}\right)=o(1 / \sqrt{n})$ w.p. 1 , since $\gamma$ is continuously differentiable. The lemma follows. 
Theorem 1. Let $t$ denote the A.P.O. rule (6). If (11) and (14) hold, then

and

$$
\rho_{0}(c)=2 V_{0} \sqrt{c}+c \int_{\Omega} \gamma^{\prime}(\theta)^{2} d \pi_{0}+o(c)
$$

$$
E\left[L_{t}(c)\right]=\rho_{0}(c)+o(c), \quad \text { as } c \rightarrow 0 .
$$

Proof. It follows directly from (9) and Lemmas 3 and 4 that

$$
\begin{aligned}
E\left[L_{t}(c)\right] & =2 \sqrt{c} E\left(\sqrt{U_{t}}\right)+E\left[r_{t}^{-1}\left(r_{t} \sqrt{c}-\sqrt{U_{t}}\right)^{2}\right] \\
& =2 \sqrt{c}\left\{V_{0}+\frac{1}{2} \sqrt{c} \int_{\Omega} \gamma^{\prime}(\theta)^{2} d \pi_{0}+o(\sqrt{c})\right\}+o(c),
\end{aligned}
$$

so that $E\left[L_{t}(c)\right]$ is given by the right side of (15).

Since $\rho_{0}(c) \leqq E\left[L_{t}(c)\right]$ for all $c>0$, it now suffices to show that $\rho_{0}(c)$ is at most as large as the right side of (15). Let $\tau=\tau_{c}$ denote the optimal stopping time - that is, $\rho_{0}(c)=E\left[L_{\tau}(c)\right], \quad c>0$. Then it follows from (7) that $\sqrt{c} \tau_{c} \rightarrow \sqrt{\psi^{\prime \prime}(\omega)}$ w.p.l as $c \rightarrow 0$; for otherwise one would have liminf $c^{-\frac{1}{2}} L_{\tau}(c) \geqq 2 \sqrt{\psi^{\prime \prime}(\omega)}$ with strict inequality on a set of positive probability, in which case Fatou's Lemma would require that $\lim$ inf $c^{-\frac{1}{2}} E\left[L_{\tau}(\psi)\right]>2 V_{0}$, contradicting $\rho_{0}(c) \leqq 2 V_{0} \sqrt{c}+O(c)$. Thus, Lemma 3 is applicable to $s=\tau$; and, when combined with (9), Lemma 3 asserts that

$$
E\left[L_{\tau}(c)\right] \geqq 2 \sqrt{c} E\left[\sqrt{U_{t}}\right] \geqq 2 \sqrt{c}\left\{V_{0}+\frac{1}{2} \sqrt{c} \int_{\Omega} \gamma^{\prime}(\theta)^{2} d \pi_{0}+o(\sqrt{c})\right\}
$$

to complete the proof of the theorem.

The limitations imposed by the theorem's conditions are illustrated by two examples.

Example 1. Suppose that $F_{\omega}$ is the exponential distribution with unknown failure rate $|\omega|$, where $-\infty<\omega<0$ : that is, suppose that $F_{\omega}$ has density $f_{\omega}(x)$ $=|\omega| \exp (\omega x)$ for $x>0$ and $-\infty<\omega<0$, w.r.t. Lebesgue measure. Then $F_{\omega}$, $-\infty<\omega<0$, form an exponential family with $\psi(\omega)=\log (1 /|\omega|)$; and the mean and variance of $F_{\omega}$ are $\theta=\psi^{\prime}(\omega)=1 /|\omega|$ and $\psi^{\prime \prime}(\omega)=1 / \omega^{2}=\theta^{2}$ for $-\infty<\omega<0$. In particular, $\gamma(\theta)=\sqrt{\psi^{\prime \prime}(\omega)}=\theta, 0<\theta<\infty$. The conjugate prior distributions are gamma distributions for $|\omega|$,

$$
\xi_{0}(\omega)=\Gamma\left(a_{0}\right)^{-1} b_{0}^{a_{0}}|\omega|^{a_{0}-1} e^{-b_{0} \omega}, \quad-\infty<\omega<0,
$$

with shape parameter $a_{0}=r_{0}+1>1$ and scale parameter $b_{0}=r_{0} \mu_{0}>0$.

Clearly, condition (11) is satisfied iff both $\theta^{2}$ and $1 / \theta^{2}$ are integrable w.r.t. $\pi_{0}$; and this is the case iff $a_{0}>2$. Condition (14) is also satisfied if $a_{0}>2$. Indeed, letting $S_{n}=X_{1}+\ldots+X_{n}$, one finds easily that

$$
U_{n}=E\left(\theta^{2} \mid \mathscr{A}_{n}\right)=\left(b_{0}+S_{n}\right)^{2} /\left(a_{0}+n-1\right)\left(a_{0}+n-2\right)
$$

and

$$
U_{n-1} / U_{n} \leqq\left(a_{0}+n-1\right) /\left(a_{0}+n-3\right)=1+2 /\left(a_{0}+n-3\right), \quad n \geqq 1
$$


Thus, Theorem 1 is applicable under the sole assumption that $a_{0}>2$, which is necessary for $\theta$ to have a finite variance. The terms in the asymptotic expansion for $\rho_{0}(c)$ may be easily computed as

$$
V_{0}=\frac{b_{0}}{a_{0}-1} \text { and } \int_{\Omega} \gamma^{\prime}(\theta)^{2} d \pi_{0}=1
$$

Analogues of this problem in continuous time have received recent attention. Shapiro and Wardrop (1980b) consider the problem when $S_{n}, n \geqq 1$, are the arrival times of a Poisson Process which is observed continuously. They show that the infinitesimal look ahead rule is optimal in this case. Rasmussen (1980) considers the problem in which the partial sums $S_{n}, n \geqq 1$, are replaced by a continuously observed gamma process with mean $\theta$ per unit time. She develops an optimal procedure which is closely related to the A.P.O. rule (6).

Example 2. If $X=0$ and 1 with probabilities $1-\theta$ and $\theta$, where $0<\theta<1$ is unknown, then the distributions of $X$ form an exponential family with $\omega$ $=\log \theta-\log (1-\theta)$ and $\psi(\omega)=\log (1 /(1-\theta))$. The mean and variance of $X$ are $\theta$ and $\psi^{\prime \prime}(\omega)=\theta(1-\theta)$; so $\gamma(\theta)=\sqrt{[\theta(1-\theta)]}, 0<\theta<1$. The conjugate prior distributions are beta distributions for $\theta$,

$$
\xi^{*}(\theta)=\frac{\Gamma\left(a_{0}+b_{0}\right)}{\Gamma\left(a_{0}\right) \Gamma\left(b_{0}\right)} \theta^{a_{0}-1}(1-\theta)^{b_{0}-1}, \quad 0<\theta<1,
$$

where $r_{0}=a_{0}+b_{0}$ and $\mu_{0}=a_{0} /\left(a_{0}+b_{0}\right)$. In this case, the conditions of Theorem 1 are satisfied if $a_{0}>1<b_{0}$; and the coefficients in the asymptotic expansion are

and

$$
V_{0}=\Gamma\left(a_{0}+\frac{1}{2}\right) \Gamma\left(b_{0}+\frac{1}{2}\right) / \Gamma\left(a_{0}\right) \Gamma\left(b_{0}\right)\left(a_{0}+b_{0}\right)
$$

$$
\int_{\Omega} \gamma^{\prime}(\theta)^{2} d \pi_{0}=\frac{1}{4}\left[\frac{b_{0}}{a_{0}-1}+\frac{a_{0}}{b_{0}-1}-2\right] .
$$

It is disappointing that Theorem 1 is not applicable to the case of a uniform prior distribution, $a_{0}=1=b_{0}$. In fact, the coefficient of $c$ in the asymptotic expansion for $\rho_{0}(c)$ diverges to $\infty$ as $a_{0} \rightarrow 1$ or $b_{0} \rightarrow 1$ from above. Alvo and Cabilio (1979) have shown that the A.P.O. rule has deficiency of order $c$ when the prior distribution is uniform, but they do not determine the coefficient of $c$.

Theorem 1 may be specialized to the Poisson and geometric cases too.

\section{The Normal Case}

In this section $X_{1}, X_{2}, \ldots$ denote random variables which are i.i.d. and normally distributed, given the common mean $\theta$ and variance $\sigma^{2}$; and $\left(\theta, \sigma^{2}\right)$ is assumed to have a conjugate prior, normal-gamma distribution. That is, the conditional distribution of $\theta$, given $\sigma^{2}$, is normal with mean $m_{0}$ and variance $r_{0}^{-1} \sigma^{2}$, where $-\infty<m_{0}<\infty$ and $r_{0}>0$; and the marginal distribution of $\sigma^{-2}$ is 
gamma with shape parameter $\frac{1}{2} a_{0}$ and scale parameter $\frac{1}{2} b_{0}$, where $a_{0}>0<b_{0}$. The posterior distribution of $\left(\theta, \sigma^{2}\right)$, given $X_{1}, \ldots, X_{n}$, is then again a normalgamma distribution with updated parameters

and

$$
a_{n}=a_{0}+n, \quad r_{n}=r_{0}+n, \quad m_{n}=\left(r_{0} m_{0}+S_{n}\right) / r_{n},
$$

$$
b_{n}=b_{0}+\sum_{i=1}^{n}\left(X_{i}-\bar{X}_{n}\right)^{2}+\frac{r_{0} n}{r_{n}}\left(\bar{X}_{n}-m_{0}\right)^{2}
$$

where $\bar{X}_{n}=S_{n} / n$ with $S_{n}=X_{1}+\ldots+X_{n}, n \geqq 1$. The problem considered is again sequential estimation of the mean $\theta$ with a $\operatorname{cost} c>0$ for each observation, but with loss $|\hat{\theta}-\theta|^{2 q}$ for estimation error, where $0<q<\frac{1}{2} a_{0}$.

As above, a decision procedure is a pair $(t, \hat{\theta})$, where $t$ is a stopping time w.r.t. $\mathscr{A}_{n}=\sigma\left\{X_{1}, \ldots, X_{n}\right\}, n \geqq 1$, and $\hat{\theta}_{t}$ is a real valued $\mathscr{A}_{t}$-measurable function; and the Bayes risk of $\left(t, \hat{\hat{\theta}_{t}}\right)$ is the expected value of $\left|\theta-\hat{\theta}_{t}\right|^{2 q}+c t$. For any stopping time $t$, the expected loss due to estimation error is minimized by letting $\hat{\theta}_{t}=m_{t}=E\left(\theta \mid \mathscr{A}_{t}\right)$, and the conditional expected loss may be written in the form

where

$$
E\left[\left|\theta-m_{t}\right|^{2 q} \mid \mathscr{A}_{t}\right]=r_{t}^{-q} K_{q} U_{t}
$$

$$
\begin{aligned}
& K_{q}=2^{q} \Gamma\left(\frac{1}{2}+q\right) / \Gamma\left(\frac{1}{2}\right), \\
& U_{n}=E\left[\sigma^{2 q} \mid \mathscr{A}_{n}\right]=C_{q}\left(a_{n}\right)\left(a_{n}^{-1} b_{n}\right)^{q}, \quad n \geq 0,
\end{aligned}
$$

and

$$
C_{q}(a)=\left(\frac{1}{2} a\right)^{q} \Gamma\left(\frac{1}{2} a-q\right) / \Gamma\left(\frac{1}{2} a\right), \quad a>2 q
$$

Let

$$
L_{n}(c)=r_{n}^{-q} K_{q} U_{n}+c r_{n} \quad n \geqq 0, c>0 .
$$

Then the Bayes risk of a sequential procedure $\left(t, m_{t}\right)$, where $t$ is a stopping time, is $E\left[L_{t}(c)\right]-c r_{0}$; so, the problem of finding a decision procedure which minimizes the Bayes risk is equivalent to finding a stopping time which minimizes $E\left[L_{t}(c)\right]$. Let

$$
\rho_{0}(c)=\inf _{s} E\left[L_{s}(c)\right], \quad c>0
$$

where the infimum extends over all stopping times $s$; and let

$$
t=t_{c}=\inf \left\{n \geqq 0: U_{n}<c\left(1 / q K_{q}\right) r_{n}^{q+1}\right\}, \quad c>0,
$$

denote the A.P.O. rule. See Bickel and Yahav (1968).

Theorem 2. Suppose $0<q<\frac{1}{2} a_{0}$. If $t$ denotes the A.P.O. rule, then

and

$$
\rho_{0}(c)=\left(\frac{q+1}{q}\right)\left(q K_{q}\right)^{\frac{1}{q+1}} V_{0} \frac{q}{c^{q+1}}+\left(\frac{q^{2}}{q+1}\right) c+o(c)
$$

where

$$
E\left[L_{t}(c)\right]=\rho_{0}(c)+o(c), \quad \text { as } c \rightarrow 0,
$$

$$
V_{0}=E\left[\sigma^{2 p}\right]=C_{p}\left(a_{0}\right)\left(a_{0}^{-1} b_{0}\right)^{p}
$$


with

$$
p=q /(1+q)
$$

Proof. For fixed $n$, a Taylor series expansion of $L_{n}(c)$ about

yields

$$
r\left(c, U_{n}\right)=c^{\frac{-1}{q+1}}\left(q K_{q} U_{n}\right)^{\frac{1}{q+1}}
$$

with

$$
L_{n}(c)=L_{n, 1}(c)+L_{n, 2}(c),
$$

and

$$
L_{n, 1}(c)=c^{\frac{q}{q+1}}\left(\frac{q+1}{q}\right)\left(q K_{q} U_{n}\right)^{\frac{1}{q+1}}
$$

$$
L_{n, 2}(c)=\frac{1}{2} q(q+1) K_{q} U_{n}\left(\frac{1}{r_{n}^{*}}\right)^{q+2}\left[r_{n}-r\left(c, U_{n}\right)\right]^{2}
$$

where $r_{n}^{*}$ denotes an intermediate point between $r_{n}$ and $r\left(c, U_{n}\right)$. The proof of Theorem 2 now proceeds along the same lines as that of Theorem 1. The details are omitted.

\section{References}

1. Alvo, M.: Bayesian sequential estimates. Ann. Statist. 5, 955-968 (1977)

2. Alvo, M., Cabilio, P.: On the sequential estimation of the probability of success. Austral. J. Statist., 21, 66-70 (1979)

3. Barndorff-Nielsen, O.: Information and Exponential Families in Statistical Theory. New York: Wiley 1978

4. Bickel, O., Yahav, J.: Asymptotically pointwise optimal procedures in sequential analysis. Proc. Fifth Berkeley Sympos. Math. Statist. Prob., 1, 401-413, University of California 1967

5. Bickel, P., Yahav, J.: Asymptotically optimal Bayes and minimax procedures in sequential estimation. Ann. Math. Statist., 39, 442-456 (1968)

6. Bickel, P., Yahav, J.: An A.P.O. rule in sequential estimation with quadratic loss. Ann. Math. Statist., 40, 417-426 (1969a)

7. Bickel, P., Yahav, J.: Some contributions to the asymptotic theory of Bayes solutions. Z. Wahrscheinlichkeitstheorie und verw. Gebiete, 11, 257-276 (1969 b)

8. Chow, Y.S., Robbins, H., Siegmund, D.: Great Expectations: the Theory of Optimal Stopping. Houghton Mifflin Co. 1970

9. Diaconis, P., Ylvisaker, D.: Conjugate priors for exponential families. Ann. Statist., 6, 269-281 (1979)

10. Ferguson, T.: Mathematical Statistics: a Decision Theoretic Approach (1967)

11. Johnson, R.: An asymptotic expansion for posterior distributions. Ann. Math. Statist. 41, 18991907 (1970)

12. Lehmann, E.: Testing Statistical Hypotheses. New York: Wiley 1959

13. Rasmussen, S.: Bayesian sequential estimation. Ann. Statist. 8, 1229-1234 (1980)

14. Shapiro, C., Wardrop, R.: Bayesian sequential estimation for one parameter exponential families. J. Amer. Statist. Ass. 75, 984-988 (1980a)

15. Shapiro, C., Wardrop, R.: Dynkin's identity applied to Bayes' sequential estimation of a Poisson process. Ann. Statist. 8, 171-180 (1980)

16. Vardi, Y.: Asymptotic optimality of certain sequential estimators. Ann. Statist. 7, 1034-1039 (1979) 